\title{
Erratum to: Biodegradation of Dichlorodiphenyltrichloroethanes (DDTs) and Hexachlorocyclohexanes (HCHsc) with Plant and Nutrients and Their Effects on the Microbial Ecological Kinetics
}

\author{
Guangdong Sun • Xu Zhang • Qing Hu • Heqing Zhang •
}

Dayi Zhang • Guanghe Li

Published online: 29 October 2014

(C) Springer Science+Business Media New York 2014

Erratum to: Microb Ecol (2014)

DOI 10.1007/s00248-014-0489-z

The original version of this article unfortunately contained mistakes in Figs. 3 and 4. The correct version is presented below:

The online version of the original article can be found at http://dx.doi.org/ 10.1007/s00248-014-0489-z.

G. Sun $\cdot$ X. Zhang $\cdot$ G. Li $(\bowtie)$

State Key Laboratory of Environmental Simulation and Pollution Control, School of Environmental Science, Tsinghua University, 100084 Beijing, China

e-mail: ligh@mail.tsinghua.edu.cn

G. Sun

e-mail: sungd11@mails.tsinghua.edu.cn

Q. $\mathrm{Hu} \cdot \mathrm{H}$. Zhang

China Energy Conservation DADI Environmental Remediation Co., Ltd, State Environmental Protection Engineering Center for Industrial Contaminated Sites and Groundwater Remediation, 100082 Beijing, China

D. Zhang

Lancaster Environment Centre, Lancaster University, Lancaster LA1

2YQ, UK 
(a)

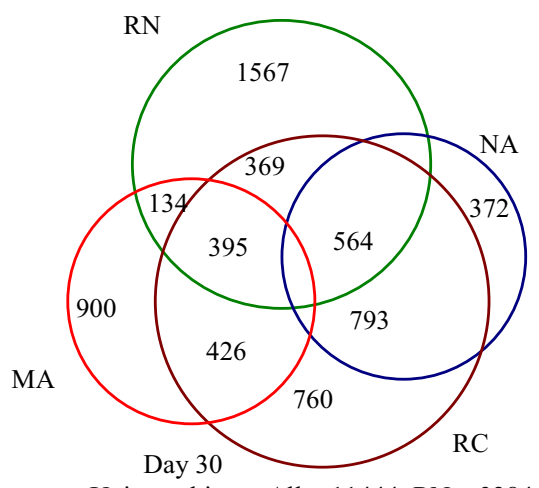

Unique objects: $\mathrm{All}=11444 ; \mathrm{RN}=3284$ $\mathrm{NA}=2324 ; \mathrm{RC}=3954 ; \mathrm{MA}=2125$ (b)

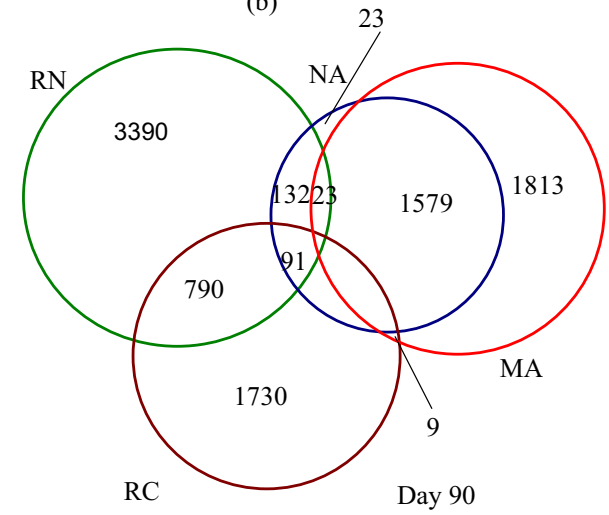

Unique objects: $\mathrm{All}=14161 ; \mathrm{RN}=4431$; $\mathrm{NA}=2321 ; \mathrm{RC}=3289 ; \mathrm{MA}=4018$

Fig. 3 Venn diagram from (a) on day 30 and (b) on day 90 pyrosequencing analysis for bacterial OTUs at $97 \%$ cutoff found in hypoliths and soil samples. Numbers of shared and unique OTUs for bacterial communities in different soil samples at different times.

Fig. 4 Shannon-Weiner (a) and equitability indices $\left(\times 10^{-1}\right)$ (b) showing diversity of bacterial communities accompanied by the change of time, as measured by differences in $16 \mathrm{~S}$ rRNA gene composition using a $3 \%$ dissimilarity cutoff. 